\section{Treatment free remission in chronic myeloid leukemia: Lights and shadows}

\author{
Matteo Molica, ${ }^{1 *}$ Nelida I. Noguera, ${ }^{2 *}$ \\ Malgorzata Monika Trawinska, ${ }^{1}$ \\ Giovanni Martinelli, ${ }^{4}$ \\ Claudio Cerchione, ${ }^{4 * *}$ \\ Elisabetta Abruzzese ${ }^{3 \text { ** }}$ \\ ${ }^{1}$ Hematology, S. Eugenio Hospital, ASL \\ Roma 2, Rome; ${ }^{2}$ Department of \\ Biomedicine and Prevention, Tor \\ Vergata University of Rome; ${ }^{3}$ Neuro \\ Onco-hematology Unit, Santa Lucia \\ Foundation, I.R.C.C.S. Rome; \\ ${ }^{4}$ Hematology Unit, Istituto Scientifico \\ Romagnolo per lo Studio e la Cura dei \\ Tumori (IRST) IRCCS, Meldola (FC), \\ Italy
}

\begin{abstract}
In addition to the best possible overall survival, discontinuation of the tyrosine kinase-inhibitor (TKI) treatment [treatment free remission (TFR)] without observing a recurrence of the disease has become a standard part of chronic myeloid leukemia (CML) care. Worldwide, more than 2000 patients with CML have attempted TFR, and very rare instances of disease transformation have been reported.
\end{abstract}

Several studies in the last decade have demonstrated the feasibility and safety of TKI discontinuation in selected patients with CML who achieve deep and sustained molecular response with TKI. This has moved prime-time into clinical practice although open questions remain in terms of understanding the disease biology that leads to successful TKI cessation in some patients while not in others. Despite the remaining questions regarding which factors may be considered predictive for TFR, treatment interruption is a safe option provided that adequate molecular monitoring is available, with prompt re-initiation of TKIs as soon as major molecular response has been lost.

Data from ongoing trials should help refine decisions as to which patients are the best candidates to attempt TKI discontinuation, frequency of a safe monitoring, optimal strategies to sustain ongoing TFR and increase the number of patients who can access to discontinuation programs.

\section{Introduction}

Tyrosine kinase inhibitors (TKIs) represent a major breakthrough in the treatment of chronic myeloid leukemia (CML). Currently, the US Food and Drug Administration (FDA) has approved four TKIs for the treatment of chronic phase CML (CP-CML) in frontline setting: imatinib, dasatinib (Sprycel, Bristol-Myers Squibb), nilotinib (Tasigna, Novartis), and bosutinib (Bosulif, Pfizer); ponatinib (Iclusig, Insight), a third generation TKI, has been approved for second and later lines. Thanks to these agents, CML changed from a disease with limited treatment options and a dismal prognosis into a more indolent disease with survival comparable to that of the general population.

The European LeukemiaNet (ELN) and European Society for Medical Oncology (ESMO) guidelines recommend basing the choice of first-line TKI on treatment goal, age and comorbidities, considering the safety profile of the TKI. ${ }^{1,2}$ Indeed, distinct safety profiles for each of imatinib, nilotinib, and dasatinib have emerged from clinical trials and clinical practice. ${ }^{3-6}$

A deep molecular response (DMR), which is identified by measuring blood $B C R$ $A B L 1$ transcript levels using real-time quantitative polymerase chain reaction (RQPCR), represents a new cutoff of molecular residual disease. MR4.0 corresponds to a $B C R / A B L 1$ ratio of less than $0.01 \%$, MR 4.5 corresponds to a ratio of less than $0.0032 \%$ and MR5.0 corresponds to a 5-log reduction, or less than $0.001 \%$ according to the International Scale. ${ }^{7}$

Evidence from several studies suggests that in patients who have achieved a sustained stable DMR, TKI treatment can be safely discontinued with close monitoring without relapse, despite $B C R / A B L 1$ DNA remaining detectable. $^{8-12}$ As a consequence, DMR yielding treatment-free remission (TFR) is increasingly regarded as a feasible treatment goal in CML, however, TFR is only successful in $40-60 \%$ of patients. ${ }^{13}$

\section{Who Is a Candidate for Treatment-free Remission?}

In the past few years, international treatment guidelines for CML have incorporated recommendations for attempting discontinuation of treatment with TKIs outside of the setting of a clinical trial with the aim of a TFR. Physicians involved in the treatment of CML need to be well informed to guide patients through decision-making about the discontinuation of treatment with TKIs targeting $B C R-A B L 1$ by providing a
Correspondence: Nelida I. Noguera, Department of Biomedicine and Prevention, Tor Vergata University of Rome, Rome, Italy and Neuro Oncohematology Unit, Santa Lucia Foundation, I.R.C.C.S. Rome, Italy.

Tel. +393341091283

E-mail: nelida.ines.noguera@uniroma2.it

Key words: chronic myeloid leukemia, tyrosine kinase inhibitors, treatment free remission.

* Matteo Molica and Nelida I. Noguera equally contributed as co-first authors

**Claudio Cerchione and Elisabetta Abruzzese equally contributed as co-last authors.

Disclosures: Elisabetta Abruzzese has served as Consultant and/or Advisory Board for Bristol Myers Squibb, Incyte, Novartis, Pfizer. None of the other authors have any conflicts of interest to declare.

This work is licensed under a Creative Commons Attribution-NonCommercial 4.0 International License (CC BY-NC 4.0).

${ }^{\circ}$ Copyright: the Author(s), 2020

Licensee PAGEPress, Italy

Hematology Reports 2020; 12(s1):8950

doi:10.4081/hr.2020.8950

balanced assessment of the potential risks and benefits of stopping or continuing therapy.

Given the heterogeneity of results reported in clinical trials and the difficulty of accurately predicting the success of TFR in individual patients, recommendations for patient criteria before stopping TKIs and for proper monitoring after discontinuation have been proposed (Table 1). ${ }^{2,13-15}$ These practice guidelines include criteria for attempting TFR outside clinical trials in order to help guide clinicians in safely and properly offering this option to selected patients. The concept of excluding patients with a history of advanced disease from possible discontinuation, as well as the molecular monitoring of the disease by a sensitive $(\geq M R 4.5)$ reverse transcription PCR standardized to the International Scale to be performed monthly in the first 6 months of TKI discontinuation, is universally shared.

According to the ESMO Clinical Practice Guidelines, prerequisites for safe stopping include: institutional requirements for safe supervision, the identification of typical $B C R$ $A B L 1$ transcripts at diagnosis, at least 5 years of TKI therapy, achievement of MR4.5, and a stable DMR (at least MR4) for at least 2 years. Notably, longer TKI therapy and longer DMR improve the stability of TFR. ${ }^{16}$ The NCCN guidelines provide the following criteria for TKI discontinuation: age $(\geq 18$ years), chronic phase CML (CP-CML; no 
prior history of accelerated or blast phase), prior TKI therapy for $\geq 3$ years, stable DMR (defined as at least MR4) for $\geq 2$ years, and unceasing molecular monitoring. The new 2020 ELN recommendations, recently published, also define minimal and optimal criteria for discontinuation. ${ }^{17}$ The optimal criteria include a DMR duration $>5$ years and a stable MR4 maintained for at least 3 years or a stable MR4.5 for at least two consecutive years. However, available recommendations are discordant at some points, and, despite several clinical trials showing TFR feasibility in CML patients with sustained DMR, the ideal selection criteria for patients in clinical practice remain uncertain, ${ }^{18}$ especially regarding the definition of DMR depth and its duration, which appear to be the paramount variables combined with a successful TFR.

\section{Mechanisms Underlying TFR Success and Relapse}

The exact mechanisms underlying successful TFR vs relapse are unclear. Several studies reported data on clinical and biologic predictive factors related to TFR success, aiming to identify the best criteria for potential discontinuation eligibility. However, to date, all of these factors are far from impacting clinical treatment policies, and a validated TFR predictive model has not yet been delineated.

Having a higher number of circulating natural killer (NK) cells was correlated with improved rates of TFR. In the DADI study, univariate analysis revealed that a higher number of total NK cells (CD3-D56+), a higher number of cytolytic NK cells (CD16+CD56+), a lower number of $\gamma \delta+\mathrm{T}$ cells, and a lower number of CD4+ regulatory $\mathrm{T}$ cells were associated with successful maintenance of TFR. ${ }^{19}$ In a subanalysis of EURO-SKI, a higher proportion of NK cells was associated with better molecular relapse-free survival, but a similar association was not found when T cells or B cells were assessed. ${ }^{20}$

As additional biologic criteria, the potential role of residual leukemic stem cells (LSC) on TFR failure has been recently investigated. Several studies have reported on the persistence of quiescent LSC in patients with CML after TKI discontinuation and after allogeneic stem cell transplant, ${ }^{21,22}$ which did not necessarily translate into relapse of disease. Interestingly, recent results have shown that flow cytometry of peripheral blood may detect residual circulating $\mathrm{CD}_{2} 6^{+} \mathrm{LSC}$ in stable TFR patients, even if with fluctuating values, with a progressive increase in relapsed patients. ${ }^{23}$ This study also documented no difference in CD26+ peripheral blood LSC between patients who relapsed or remained in remission after TKI discontinuation.

As clinical criteria, total duration of TKI therapy and DMR are the most consistently reported predictive factors for achieving a TFR. In the Stop Imatinib trial (STIM), longterm follow-up showed that patients with CML who received imatinib for more than 50 months experienced a lower rate of molecular relapses than those who received imatinib for less time. ${ }^{24}$ In the EURO-SKI trial, an interim analysis of 405 patients who received imatinib as first-line treatment demonstrated that longer treatment duration and longer DMR durations were associated with increasing probability of MMR maintenance at 6 months. ${ }^{25}$

In the imatinib suspension and validation (ISAV) study, both age ( $<45$ years) and a positive digital PCR status were shown to predict relapse in CML patients who

Table 1. Criteria for Discontinuing TKIs Based on Expert Recommendations and Guidelines

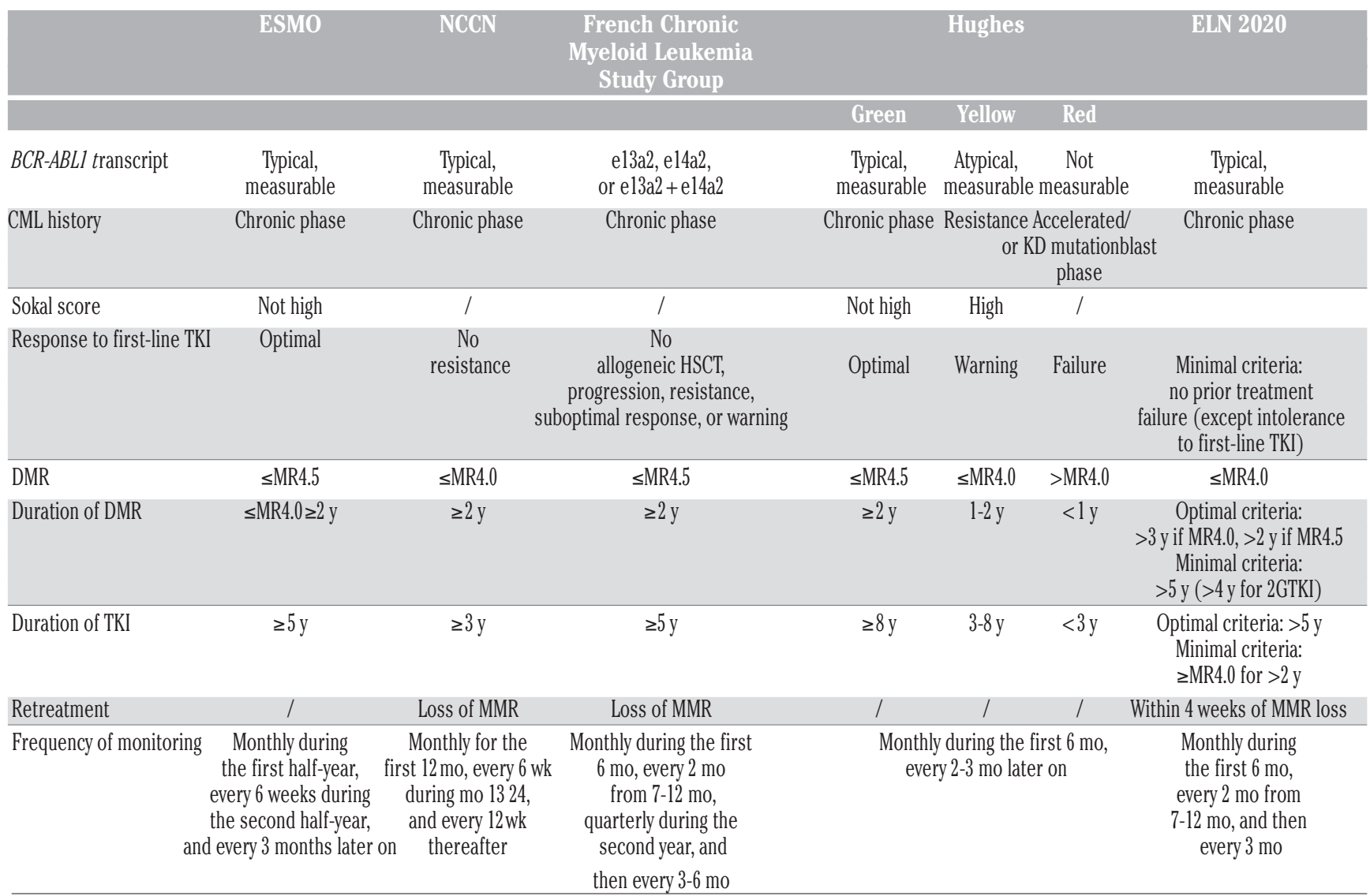

CML, chronic myeloid leukemia; DMR, deep molecular response; ESMO, European Society for Medical Oncology; HSCT, hematopoietic stem cell transplant; MMR, major molecular response; mo, months; MR4.0, $B C R A B L 1$ ratio $<0.01 \%$; MR4.5, BCR/ABLI ratio <0.0032\%; TKI, tyrosine kinase inhibitor; wk, weeks; y, years. 
discontinued imatinib. ${ }^{26}$ However, the DASFREE study reported older age as a favorable prognostic factor of 2-years TFR in patients with CML. ${ }^{27}$ According to these results, current recommendations do not consider age as a limiting factor for discontinuation eligibility. ${ }^{28}$ Digital PCR is 100 times more sensitive than RQ-PCR. Although it is not yet routinely standardized for detecting molecular MRD in CML, preliminary data suggest that it could be more accurate for TFR prediction. ${ }^{29}$ In a recent Italian study, investigators reported that digital PCR was able to identify patients at elevated risk for molecular response loss after discontinuation. Furthermore, the TFR rate of patients who discontinued TKI with a digital PCR of less than 0.468 was significantly higher compared with patients with a digital PCR of at least 0.468 (TFR at 2 years was $83 \%$ vs $52 \%$, respectively; $P=.0017){ }^{30}$

The potential predictive role of $B C R$ $A B L$ transcript type (e13a2 and e14a2) in achieving a sustained DMR and TFR was reported in several studies, as recently reviewed. ${ }^{31}$ Despite a direct association with outcome is still controversial, a higher incidence of sustained DMR and TFR success was shown for patients harboring the e14a2 transcript vs. e13a2, possibly due to differences in immunogenicity, technical or genetic reasons. ${ }^{32,33}$

Sokal risk score has been also identified as an important prognostic factor for successful TFR with imatinib. ${ }^{11,34}$ Data from TWISTER study suggested that a high $\square$ risk Sokal score at diagnosis was associated with molecular relapse (34). Mahon et al. reported that patients from STIM with low $\square$ risk Sokal scores had an estimated survival without relapse at 18 months of $54 \%$, compared with $35 \%$ and $13 \%$ in those with intermediate and high scores, respectively. ${ }^{13}$

\section{Most important trials including TFR}

Many studies, some retrospective and some prospective, have shown beyond any doubt that a consistent proportion, ranging between $30 \%$ and $70 \%$, of the patients who discontinue treatment after having achieved a DMR may remain treatment-free for an asyet undefined period of time.

\section{Imatinib}

The French STIM trial first selected patients on imatinib therapy for a minimum of 3 years with undetectable transcripts (with an RQ $\square$ PCR sensitivity of at least 5 logs) sustained for more than 2 consecutive years, and with at least 5 assessments during those 2 years. ${ }^{33}$ After a follow-up of 77 months after discontinuation of therapy, the molecular relapse-free survival rate was $43 \%$ at 6 months and $38 \%$ at 60 months, with the majority of relapses occurring within 6 months after imatinib cessation. The TWISTER study, from the Australasian Leukaemia Lymphoma Group, used a similar study design. This Australasian CML8 trial enrolled patients who had received 3 years of imatinib therapy and had undetectable minimal residual disease (UMRD) for 2 years (UMRD was confirmed before enrollment in a central laboratory with 4.5- $\log$ assay sensitivity. ${ }^{34}$ With a median follow-up of 42 months, molecular recurrence was reported in 22 of 40 patients; the estimated TFR rate at 24 months was $47.1 \%$. Data were recently published from the EURO-SKI study, the largest trial on TFR to date. This trial included 758 patients (94\% treated with first-line imatinib) with at least 3 years of TKI treatment and MR4.0 sustained for at least 1 year. After a median follow-up of 27 months, molecular relapsefree survival was $61 \%$ at 6 months and $50 \%$ at 24 months. Of the 758 patients, 371 (49\%) lost MMR after TKI discontinuation. ${ }^{35}$ Recently, a meta-analysis was reported from 15 different cohort studies, involving 509 patients who stopped imatinib in molecular response (MR), including the STIM and ASTIM studies. ${ }^{36}$ The overall rate of molecular relapse was $51 \%$; after 6 months of follow-up, it was $41 \%$, confirming that $80 \%$ of molecular relapses occurred in the first 6 months.

\section{Nilotinib and dastinib}

ENESTfreedom was a single-arm, phase 2 trial which assessed the potential for TFR in patients with CP-CML who had $\geq 2$ years of frontline nilotinib treatment and sustained DMR during a 52-weeks nilotinib consolidation phase (defined as MR4.5 in the last assessment, no assessment worse than MR4, and $\leq 2$ assessments between MR4 and MR4.5). ${ }^{37}$ At 192 weeks, the TFR rate was $44.2 \%$ (84 of 190 patients), MMR and $\mathrm{MR}^{4.5}$ were regained by $98.9 \%$ (90 of 91) and $92.3 \%$ (84 of 91) of patients, respectively, who resumed nilotinib. Nilotinib discontinuation was also evaluated as second-line treatment in the ENESTop trial. The study enrolled patients treated with imatinib for more than 3 years with detectable molecular response who switched to nilotinib for more than 2 years (51 switched owing to intolerance, 30 switched owing to resistance, and 44 switched owing to physician preference) and discontinued when stable MR4.5 was reached. A total of 126 patients discontinued nilotinib in
ENESTop; $58 \%$ remained off therapy at week 48 and $53 \%$ continued to remain off therapy at week 96 of the TFR phase. ${ }^{38}$

In the 2-years update of the DASFREE study, including 84 patients with CP-CML and sustained DMR, TFR at 2 years following dasatinib discontinuation was $46 \% .{ }^{39}$ In this study, patients were eligible to attempt TFR if they had received $\geq 2$ years of dasatinib treatment and had dasatinibinduced $\mathrm{MR}^{4.5}$ for $\geq 1$ year prior to study entry. Prognostic factors related to maintaining TFR at 2 years were age, duration of prior dasatinib, and prior therapy line. In the DADI study, patients with CPCML who received dasatinib following resistance to or intolerance of imatinib and had a $B C R / A B L 1$ value of less than $0.0069 \%$ for at least 1 year were eligible to attempt TFR. Among 63 patients who discontinued dasatinib, TFR rates were $49 \%$ at 6 months and $44 \%$ at 36 months, but the criterion for molecular relapse (defined as $B C R / A B L 1$ $\geq 0.0069 \%$ ) was more stringent than in other studies. ${ }^{40}$

\section{Real-life experiences}

In a Spanish retrospective study including 236 CP-CML patients who discontinued TKIs outside of clinical trials (patients had received TKI for $\geq 3$ years with sustained MR4.5 in $\geq 4$ consecutive assessments over a minimum of 2 years prior to discontinuation). ${ }^{41}$ treatment-free survival at 4 years was $64 \%$; no cases of disease progression were observed and, in patients who failed TFR, a DMR was regained 3-5 months after treatment re-introduction. A shorter duration of both TKI treatment $(<5$ years) and duration of MR4.5 before discontinuation $(<4$ years) were associated with significantly increase the risk of molecular relapse. In a retrospective analysis of 293 Italian patients with CP-CML in DMR who stopped treatment with various TKIs. ${ }^{42}$ patients discontinuing secondgeneration TKIs had a median duration of treatment with the last TKI of 50 vs. 96 months of treatment with imatinib. Furthermore, using multivariate Cox regression model, a better probability of TFR for patients treated with second-generation TKIs, with an estimated $57 \%$ relative risk reduction vs. imatinib, was observed.

\section{Second Discontinuation Attempt}

RE-STIM (Second Tyrosine Kinase Inhibitor Discontinuation Attempt in Patients With Chronic Myeloid Leukemia), a French multicenter study, evaluated TFR in 70 patients who reattempted TKI discontinuation after a first unsuccessful attempt. All the patients had re-achieved a sustained MR4.5 ( $\geq 2$ years) before 
attempting TFR the second time. TFR rates at 12,24 , and 36 months were $48 \%, 42 \%$, and $35 \%$, respectively. A more recent analysis of $106 \mathrm{CP}-\mathrm{CML}$ patients with 41 months of follow-up from the RE-STIM study confirmed the safety and success of a second TKI discontinuation attempt. ${ }^{43}$ Major factors significantly associated with TFR outcome were the speed of molecular relapse after the first TKI discontinuation and TKIfree duration of $>6$ months after the first attempt at TKI discontinuation. In a Canadian discontinuation trial called TRAD (Treatment-Free Remission Accomplished With Dasatinib in Patients With CML), 25 of the 51 patients receiving dasatinib achieved MR4.5 for 12 months or longer and discontinued it for a second TFR attempt (TFR2). The estimated TFR2 rate was $21.5 \%$ $( \pm 8.5 \%)$ at 6 months. The 6-month TFR2 rate was $9 \%$ in the group that relapsed within 3 months of TFR 1 and $30 \%$ in the group that relapsed within 3 to 6 months of TFR 1 $(\mathrm{P}<.001) .{ }^{44}$ These data suggest that more strict criteria should be considered for a TFR2 attempt, probably including achievement of deeper BCR/ABL1 levels (MR5.0) prior to the second TKI discontinuation.

\section{The advantages and disadvan- tages of TFR}

The milestones of TFR include improving quality of life, ${ }^{45}$ decreasing TKIrelated toxicities, minimizing the adverse events and economic factors associated with lifelong therapy, ${ }^{46-48}$ and preventing potential toxicities in selected setting, such as female patients of childbearing potential and adolescents. ${ }^{49}$

Different studies have shown that prolonged TKI treatment may lead to adverse effects that negatively affect quality of life. Patients who are younger or female and receiving long $\square$ term therapy with imatinib have reported a worse quality of life compared with healthy individuals. ${ }^{45}$ However, as part of the ENESTfreedom trial, patients completed questionnaires that addressed their quality of life while discontinuing first $\square$ line nilotinib. Interestingly, similar levels of anxiety and depression were reported before and after treatment discontinuation. ${ }^{37}$

Long-term treatment in patients with CML can lead to TKI-related complications that may cause morbidity or mortality and the possibility of mitigating them may persuade some patients to pursue TFR. Notable adverse events associated with dasatinib are increased pulmonary arterial hypertension and pleural effusion. ${ }^{47}$ Additionally, increased peripheral arterial occlusive disease has been observed with several TKIs, especially nilotinib. ${ }^{46}$ The possibility to minimize the risk of developing new toxicities from long-term TKI treatment is one of the paramount benefit of attempting TFR.

Economic considerations may play a key role in a patient's decision to attempt TFR. The annual cost of treatment per patient, which is currently estimated at $€ 30,000$ to $€ 40,000$ in most European countries ${ }^{50}$ and more than $\$ 100,000$ in the United States. ${ }^{48}$ These costs may be somewhat offset by TFR. In a study of data derived from the ENESTnd trial, there was an incremental cost-effectiveness ratio of $€ 39,935$ per quality-adjusted life-year for patients receiving nilotinib. ${ }^{51}$ TKI discontinuation had a large economic impact in the EUROSKI trial. ${ }^{52}$ with an estimated savings of $€ 22$ million just for the 755 patients in this study.

Female patients of childbearing potential may particularly benefit from TFR as a treatment goal, because the use of TKIs during pregnancy has been associated with adverse outcomes. ${ }^{53,54}$ Preclinical studies and a review of clinical cases have shown that dasatinib interfered with normal pregnancy, resulting in fetal abnormalities. ${ }^{55}$ Although anecdotal reports have reported instances of patients with uneventful pregnancies despite continuous administration of TKIs, this practice currently is not recommended. ${ }^{56}$ Although CML is rare in the pediatric population, the most important concern in treating these patients is the safety of prolonged TKI treatment. Successful TFR in a small number of pediatric patients has been reported, ${ }^{57}$ but further studies are needed to clarify whether the TFR approach should be included in clinical practice for pediatric CML.

TFR attempt may also subtend several shadows. It should be noted that many patients are unwilling to halt treatment. In one survey, only $42 \%$ of respondents were willing to stop TKI treatment due to fears of disease recurrence and increased mortality. ${ }^{58}$ In this single $\square$ institution study, $26 \% \square 62 \%$ of patients were willing to accept discontinuation of their TKI, depending upon the perceived success rate of cessation, and the risks of disease recurrence and increased mortality.

TKI withdrawal syndrome (TKI WS), which consists of diffuse myalgia, arthralgia, or musculoskeletal pain, all of which respond to steroids or other analgesics, has been reported in patients shortly after stopping TKIs. ${ }^{59}$ In the ENESTfreedom study, $34 \%$ of patients reported musculoskeletal pain-related events during the 1st year of the TFR phase, decreasing to 9,3 , and $3 \%$ during the $2 \mathrm{nd}, 3 \mathrm{rd}$, and 4 th year. $^{37}$ In the DASFREE study, musculoskeletal events were reported in 23 (27\%) patients off treatment, although only eight events were attributed to TKI withdrawal. ${ }^{27}$ In a systematic review on the efficacy of TKI discontinuation (10 studies, $n=1,601$ patients), the weighted mean incidence of TKI WS was $27 \%$, with TKI WS occurring in the early TFR phase. ${ }^{60}$ In an analysis of 427 CML patients from the combined cohort of STIM2 and EURO-SKI trials, ${ }^{61}$ longer duration of TKI treatment and history of osteoarticular symptoms appeared to be risk factors that predispose patients to TKI WS. A better understanding of the pathophysiology of this complication is needed in order to design strategies that mitigate symptoms and the need to resume therapy. Cognitive dysfunction has also been reported following the discontinuation of TKIs in patients with CML, highlighting the need to supervise on any unexplained neurological occurrences. ${ }^{62}$

There is a consistent risk of relapse when patients attempt TFR, although relapse has been defined differently in several trials, from loss of MR4.5 to loss of MMR. The relapse rate at 6 months has ranged from $61 \%$ in the STIM trial and $49 \%$ in the EURO $\square$ SKI trial. ${ }^{33,35}$ This risk is crucial for patient's decision to attempt treatment discontinuation; in a survey of more than 1100 patients with CML, 49\% responded they would not discontinue treatment for fear of losing the response they had achieved at the time of the survey. ${ }^{63}$

The potential occurrence of sudden blast crisis should also be mentioned. In the A $\square$ STIM trial, one patient experienced a lymphoid blast crisis 9 months after restarting imatinib while in MMR. ${ }^{24}$ Recently, a case was reported in the STOP2-Generation TKIs trial ${ }^{64}$ and in a CP-CML patient who attempted dasatinib discontinuation under clinical trial conditions (NCT00254423). ${ }^{65}$ These rare events required further biological evaluation and as well as prospective assessment in real-life, although with adequate follow-up of $5 \square 6$ years, this risk appears to be minimal. To this regard an international trial has just started; it is a retrospective and prospective observational study on risk of progression in CP-CML patients eligible for tyrosine kinase inhibitor discontinuation (named TFR-PRO). The trial aim is to quantify the risk of progression to AP/BP after TKI discontinuation in CML patients who undergo a first or subsequent discontinuation attempt and is expected to monitor at least 3000 patients in 5 different countries. Also progression free and overall 
survival; incidence of Serious Adverse Events and evaluation of Quality of Life indicators will be recorded and compared in a similar patient population not discontinuing treatment.

Whether patients who relapse on TFR should re-attempt TKI discontinuation is currently unknown. Some data have shown that attempting a second TKI discontinuation after molecular recurrence is possible, and this might be effective in approximately $30 \%$ of cases after an adequate duration of the reachieved DMR. ${ }^{43,44}$ However, the rate of successful TFR in this setting remains low.

\section{Future perspectives}

Several challenges remain considering incorporation of TFR in CML scenario. TFR appears to be successful in a small subset of patients. In fact, based on the strict molecular criteria for discontinuation (MR4-4.5 sustained for $\geq 2$ years), only $40 \%$ of patients receiving TKIs may attempt TFR. Among these, approximately $50 \%$ maintained molecular responses without re-starting TKIs, and only $20 \%$ remained in TFR. Novel treatment approaches that include immunotherapy-based combination treatment and the combination of TKIs with other agents might determine the eradication or the control of the leukemic stem cell hereby increasing the rate of durable DMR. As consequence, a higher number of patients potentially will be eligible for discontinuation, with a lower rate of molecular relapse after stopping therapy.

The rationale for using IFN- $\alpha$ associated with TKIs in order to anticipate and increase deep molecular responses before TKI discontinuation was initially demonstrated by Burchert et al. ${ }^{66}$ Indeed, the combination of IFN- $\alpha$ plus TKI showed significantly higher rates of molecular responses detected in patients treated with imatinib plus IFN- $\alpha$ or with PEGylated IFN (Peg-IFN) alpha-2a compared with imatinib alone. ${ }^{67,68}$ Similarly, the combination of dasatinib followed by addition of Peg-IFN alpha-2b after 3 months determined promising results in a phase 2 trial of $40 \mathrm{CP}-\mathrm{CML}$ patients. ${ }^{69}$ An interim analysis of the PETALS study, which assessed cumulative rates of MR4.5 after 12 months of treatment with nilotinib or nilotinib plus IFN in 200 newly diagnosed CP-CML patients, showed significant higher DMR rates in the combination treatment arm at 12 months. ${ }^{70}$ Similarly, an interim results of the PINNACLE study also reported that combination therapy with nilotinib plus PegIFN alpha-2b results in favorable rates of molecular responses compared with nilotinib alone. ${ }^{71}$ Data on TFR after interferon and TKIs combination strategies are still under investigation. Other promising combinations and ongoing trials in CML patients include venetoclax in combination with dasatinib (NCT02689440) which potentially might determine higher rates of DMR and therefore increasing the number of patients potentially eligible for TFR.

Dose reductions are also being considered in clinical trials prior to TFR attempts. Dose modification of TKI therapy aims to reduce adverse events in CML patients whilst maintaining efficacy. Clinical trials such as DESTINY ${ }^{72}$ have demonstrated that dose reduction is safe for patients in deep molecular remission and may be considered prior to a TFR attempt. Clinical trials of dose optimization are currently recruiting across all approved TKIs and will lead to further personalization of therapy for CP-CML patients in the future.

Among challenges of TFR, it is also included the better identification of patients who have a high probability of relapsing compared with those who experience a successful TFR. Some of the most promising data involve the field of immunology, especially T-cell cytotoxicity and NK cell effects. In several studies, NK cell numbers or functional subsets of NK cells were shown to be higher in patients who maintain TFR than in those who lose molecular response during TKI discontinuation. ${ }^{10,73,74 .}$

The development of new molecular techniques able to better detect low levels of $B C R / A B L 1$ transcript may also improve the selection of CML patients who would likely benefit from a TFR attempt. In last years, digital PCR seemed to determine a more sensitive and accurate detection of very low levels of minimal residual disease ${ }^{29,30}$ and, therefore, in the future it could become the main monitoring technique for selecting the best candidates for TFR

\section{References}

1. Baccarani M, Deininger MW, Rosti G, et al. European LeukemiaNet recommendations for the management of chronic myeloid leukemia: 2013. Blood. 2013: 122:872-84.

2. Hochhaus A, Saussele S, Rosti G, et al. Chronic myeloid leukaemia: ESMO clinical practice guidelines for diagnosis, treatment and follow-up. Ann Oncol. 2017; 28: iv41-51.

3. Cortes JE, Saglio G, Kantarjian HM, et al. Final 5-year study results of DASISION: the dasatinib versus imatinib study in treatment-naive chronic myeloid leukemia patients trial. J Clin Oncol. 2016; 34:2333-40.

4. Hochhaus A, Saglio G, Hughes TP, et al. Long-term benefits and risks of frontline nilotinib vs imatinib for chronic myeloid leukemia in chronic phase: 5-year update of the randomized ENESTnd trial. Leukemia. 2016; 30:1044-54.

5. Steegmann JL, Baccarani M, Breccia M, et al. European LeukemiaNet recommendations for the management and avoidance of adverse events of treatment in chronic myeloid leukaemia. Leukemia. 2016; 30:1648-71.

6. Minami M, Arita T, Iwasaki H, et al. Comparative analysis of pulmonary hypertension in patients treated with imatinib, nilotinib and dasatinib. Br J Haematol. 2017; 177:578-87.

7. Cross NC, White HE, Muller MC, et al. Standardized definitions of molecular response in chronic myeloid leukemia. Leukemia. 2012; 26:2172-5.

8. Branford S, Yeung DT, Ross DM, et al. Early molecular response and female sex strongly predict stable undetectable BCR-ABL1, the criteria for imatinib discontinuation in patients with CML. Blood. 2013; 121:3818-24.

9. Hochhaus A, Masszi T, Giles FJ, et al. Treatment-free remission following frontline nilotinib in patients with chronic myeloid leukemia in chronic phase: results from the ENEST freedom study. Leukemia. 2017; 31:1525-31.

10. Imagawa J, Tanaka H, Okada M, et al. Discontinuation of dasatinib in patients with chronic myeloid leukaemia who have maintained deep molecular response for longer than 1 year (DADI trial): a multicentre phase 2 trial. Lancet Haematol. 2015; 2:e528-35.

11. Mahon FX, Rea D, Guilhot J, Guilhot F, et al. Discontinuation of imatinib in patients with chronic myeloid leukaemia who have maintained complete molecular remission for at least 2 years: the prospective, multicentre Stop Imatinib (STIM) trial. Lancet Oncol. 2010; 11:1029-35.

12. Rea D, Nicolini FE, Tulliez M, et al. Discontinuation of dasatinib or nilotinib in chronic myeloid leukemia: interim analysis of the STOP 2G-TKI study. Blood. 2017; 129:846-54.

13. Hughes TP, Ross DM. Moving treatment-free remission into mainstream clinical practice in CML. Blood. 2016; 128:17-23.

14. Radich JF, Deininger M, Abboud CN, et al. NCCN clinical practice guidelines in oncology: chronic myeloid leukemia. Hematol Oncol Clin North Am. 2017; 17:1159-1173. 
15. Rea D, Ame S, Berger $M$, et al. Discontinuation of tyrosine kinase inhibitors in chronic myeloid leukemia: Recommendations for clinical practice from the French Chronic Myeloid Leukemia Study Group. Cancer. 2018; 124:2956-2963.

16. Saussele S, Richter J, Guilhot J, et al. Discontinuation of tyrosine kinase inhibitor therapy in chronic myeloid leukaemia (EURO-SKI): a prespecified interim analysis of a prospective, multicentre, non-randomised, trial. Lancet Oncol. 2018; 19:747-57.

17. Hochhaus A, Baccarani M, Silver RT, et al. European LeukemiaNet 2020 recommendations for treating chronic myeloid leukemia. Leukemia 2020; 34 966-984.

18. Rea D, Cayuela JM. Treatment-free remission in patients with chronic myeloid leukemia. Int J Hematol. 2018; 108:355-64.

19. Imagawa J, Tanaka H, Okada M, et al. DADI Trial Group. Discontinuation of dasatinib in patients with chronic myeloid leukaemia who have maintained deep molecular response for longer than 1 year (DADI trial): a multicentre phase 2 trial. Lancet Haematol 2015; 2:e528-e535.

20. Ilander M, Olsson-Strömberg $U$, Schlums $\mathrm{H}$, et al. Increased proportion of mature NK cells is associated with successful imatinib discontinuation in chronic myeloid leukemia. Leukemia. 2017; 31:1108-1116.

21. Kaeda J, O'Shea D, Szydlo RM, et al. Serial measurement of BCR-ABL transcripts in the peripheral blood after allogeneic stem cell transplantation for chronic myeloid leukemia: an attempt to define patients who may not require further therapy. Blood. 2006; 107:41716.

22. Radich JP, Gooley T, Bryant E, et al. The significance of bcr-abl molecular detection in chronic myeloid leukemia patients "late," 18 months or more after transplantation. Blood. 2001; 98:1701-7.

23. Bocchia M, Sicuranza A, Pacelli P, Pregno $\mathrm{P}$, Annunziata $\mathrm{M}$, Sorà $\mathrm{F}$, et al. Prospective monitoring of peripheral blood CD26+ leukemia stem cells in chronic myeloid leukemia patients from time of TKI discontinuation. Blood. 2019; 134:2919.

24. Etienne G, Guilhot J, Rea D, et al. Long-term follow-up of the French Stop Imatinib (STIM1) study in patients with chronic myeloid leukemia. J Clin Oncol. 2017; 35:298-305.

25. Mahon FX, Richter R, Guilhot J, et al. Interim Analysis of a Pan European Stop
Tyrosine Kinase Inhibitor Trial in Chronic Myeloid Leukemia: The EURO-SKI study. Blood. 2014; 124:151.

26. Mori S, Vagge E, le Coutre P, et al. Age and $\mathrm{dPCR}$ can predict relapse in $\mathrm{CML}$ patients who discontinued imatinib: the ISAV study. Am J Hematol. 2015; 90:910-4.

27. Shah NP, Garcia-Gutierrez V, JimenezVelasco A, et al. Dasatinib discontinuation in patients with chronicphase chronic myeloid leukemia and stable deep molecular response: the DASFREE study. Leuk Lymphoma. 2019; 61:650-969.

28. Baccarani M, Abruzzese E, Accurso V, et al. Managing chronic myeloid leukemia for treatment-free remission: a proposal from the GIMEMA CML WP. Blood Adv. 2019; 3:4280-90.

29. Bernardi S, Ruggieri G, Malagola M, et al. Digital PCR (Dpcr) a Step Forward to Detection and Quantification of Minimal Residual Disease (MRD) in Ph+/BCR-ABL1 Chronic Myeloid Leukemia (CML). J Mol Biomark Diagn. 2017; 08:1-3.

30. Bernardi S, Malagola M, Zanaglio C, et al. Digital PCR improves the quantitation of DMR and the selection of CML candidates to TKIs discontinuation. Cancer Med. 2019.

31. Baccarani M, Rosti G, Soverini S. Chronic myeloid leukemia: the concepts of resistance and persistence and the relationship with the BCR-ABL1 transcript type. Leukemia. 2019; 33:2358-64.

32. Jain P, Kantarjian H, Patel KP, et al. Impact of BCR-ABL transcript type on outcome in patients with chronic-phase CML treated with tyrosine kinase inhibitors. Blood. 2016; 127:1269-75.

33. Rousselot P, Huguet F, Réa D, et al. Imatinib mesylate discontinuation in patients with chronic myelogenous leukemia in complete molecular remission for more than 2 years. Blood. 2007;109(1):58-60.

34. Ross DM, Branford S, Seymour JF, et al. Safety and efficacy of imatinib cessation for CML patients with stable undetectable minimal residual disease: results from the TWISTER study. Blood. 2013;122(4):515-522.

35. Saussele S, Richter J, Guilhot FX, et al. Discontinuation of tyrosine kinase inhibitor therapy in chronic myeloid leukaemia (EURO-SKI): a prespecified interim analysis of a prospective, multicentre, non-randomised, trial. Lancet Oncol. 2018; 19:747-757.

36. Campiotti L, Suter MB, Guasti L, et al.
Imatinib discontinuation in chronic myeloid leukaemia patients with undetectable BCR-ABL transcript level: a systematic review and a meta-analysis. Eur J Cancer. 2017; 77:48-56.

37. Ross DM, Masszi T, Casares GMT, et al. Durability and impact on quality of life of treatment-free remission (TFR) in patients with chronic myeloid leukemia (CML) after stopping frontline (11) nilotinib: ENESTfreedom 192-wk results. EHA 2019; Abstract PF409.

38. Hughes TP, Boquimpani CM, Benyamini N, et al. Treatment-free remission in patients with chronic myeloid leukemia in chronic phase according to reasons for switching from imatinib to nilotinib: subgroup analysis from ENESTop. Blood. 2016; 128:792.

39. Shah NP, Garcia-Gutierrez V, JimenezVelasco A, et al. Dasatinib discontinuation in patients with chronicphase chronic myeloid leukemia and stable deep molecular response: the DASFREE study. Leuk Lymphoma. 2019; 61:650-969.

40. Okada, M, Imagawa J, Tanaka H, et al. Final 3-year Results of the Dasatinib Discontinuation Trial in Patients With Chronic Myeloid Leukemia Who Received Dasatinib as a Second-line Treatment. Clin Lymphoma Myeloma Leuk. 2018; 18:353-360.

41. Hernandez-Boluda JC, Pereira A, PastorGalan I, et al. Feasibility of treatment discontinuation in chronic myeloid leukemia in clinical practice: results from a nationwide series of 236 patients. Blood Cancer J. 2018; 8:91.

42. Fava C, Rege-Cambrin G, Dogliotti I, et al. Observational study of chronic myeloid leukemia Italian patients who discontinued tyrosine kinase inhibitors in clinical practice. Haematologica. 2019; 104:1589-96.

43. Legros L, Nicolini FE, Etienne G, et al. The TKI-free duration after a first discontinuation attempt that failed in $\mathrm{CP}$ CML patients is a predictive factor of TKI-free remission after a second attempt. Blood. 2019; 134:28.

44. Kim DDH, Busque L, Forrest DL, et al. Second Attempt of TKI Discontinuation with Dasatinib for Treatment-Free Remission after Failing First Attempt with Imatinib: Treatment-Free Remission Accomplished By Dasatinib (TRAD) Trial. Oral and Poster Abstracts; ASH 2018.

45. Efficace F, Baccarani M, Breccia M, et al. Health-related quality of life in chronic myeloid leukemia patients receiving long-term therapy with imatinib compared with the general 
population. Blood. 2011; 118(17):4554-4560.

46. Damrongwatanasuk R and Fradley MG. Cardiovascular complications of targeted therapies for chronic myeloid leukemia. Curr Treat Options Cardiovasc Med. 2017; 19:24.

47. Cortes JE, Jimenez CA, Mauro MJ, et al. Pleural effusion in dasatinib-treated patients with chronic myeloid leukemia in chronic phase: identification and management. Clin Lymphoma Myeloma Leuk. 2017; 17:78-82.

48. Kantarjian HM, Fojo T, Mathisen M, Zwelling LA. Cancer drugs inthe United States: justum pretium - the just price. J Clin Oncol. 2013; 31(28):3600-3604.

49. de Bruijn CMA, Millot F, Suttorp M, et al. Discontinuation of imatinib in children with chronic myeloid leukaemia in sustained deep molecular remission: results of the STOP IMAPED study. $\mathrm{Br}$ J Haematol. 2019; 185(4):718-724.

50. Hochhaus A. Educational session: managing chronic myeloid leukemia as a chronic disease. Hematology Am Soc Hematol Educ Program 2011; 2011:128-135.

51. Aiello A, Daniel F, D'Ausilio A, et al. Cost-effectiveness of nilotinib in patients with chronic myeloid leukaemia eligible to start the treatment-free remission phase in Italy. Value Health. 2015; 18: A457. Abstract PCN153.

52. Saussele S, Richter J, Guilhot FX, et al. Discontinuation of tyrosine kinase inhibitor therapy in chronic myeloid leukaemia (EURO-SKI): a prespecified interim analysis of a prospective, multicentre, non-randomised, trial. Lancet Oncol. 2018; 19:747-757.

53. Ault P, Kantarjian H, O'Brien S, et al. Pregnancy among patients with chronic myeloid leukemia treated with imatinib. J Clin Oncol. 2006; 24(7): 1204- 1208.

54. Pye SM, Cortes J, Ault P, et al. The effects of imatinib on pregnancy outcome. Blood. 2008; 111(12): 55055508 .

55. Cortes JE, Abruzzese E, Chelysheva E, et al. The impact of dasatinib on pregnancy outcomes. Am J Hematol. 2015; 90(12): 1111- 1115.

56. Abruzzese E, Trawinska MM, Perrotti AP, De Fabritiis P. Tyrosine kinase inhibitors and pregnancy. Mediterr J Hematol Infect Dis. 2014; 6(1): e2014028

57. Giona F, Saglio G, Moleti ML, et al.
Treatment-free remission after imatinib discontinuation is possible in paediatric patients with chronic myeloid leukaemia. $\mathrm{Br}$ J Haematol. 2015; 168:305-308.

58. Goldberg S, Hamarman S. Patients with chronic myelogenous leukemia may not want to discontinue tyrosine kinase inhibitor therapy. Blood. 2015; 126: 1584.

59. Hughes TP, Ross DM. Moving treatment-free remission into mainstream clinical practice in CML. Blood. 2016; 128(1): 17- 23.

60. Chen KK, Du TF, Xiong PS, et al. Discontinuation of tyrosine kinase inhibitors in chronic myeloid leukemia with losing major molecular response as a definition for molecular relapse: a systematic review and meta-analysis. Front Oncol. 2019; 9:372.

61. Berger MG, Pereira B, Rousselot P, et al. Longer treatment duration and history of osteoarticular symptoms predispose to tyrosine kinase inhibitor withdrawal syndrome. Br J Haematol. 2019; 187:337-46.

62. Claudiani S, Apperley JF, Deplano S, et al. Cognitive dysfunction after withdrawal of tyrosine kinase inhibitor therapy in chronic myeloid leukaemia. Am J Hematol. 2016; 91:E480-E1.

63. Breccia M, Efficace F, Sica S, et al. Adherence and future discontinuation of tyrosine kinase inhibitors in chronic phase chronic myeloid leukemia: a patient-based survey on 1133 patients. Leuk Res. 2015; 39(10): 1055- 1059.

64. Rea D, Nicolini FE, Tulliez M, et al. Prognostication of molecular relapses after dasatinib or nilotinib discontinuation in chronic myeloid Leukemia (CML): a FI-LMC STOP 2GTKI study update. Blood. 2019; 134:30.

65. Alfayez M, Richard-Carpentier G, Jabbour E, et al. Sudden blastic transformation in treatment-free remission chronic myeloid leukaemia. Br J Haematol. 2019; 187:543-5.

66. Burchert A, Muller MC, Kostrewa P, et al. Sustained molecular response with interferon alfa maintenance after induction therapy with imatinib plus interferon alfa in patients with chronic myeloid leukemia. J Clin Oncol. 2010; 28:1429-35.

67. Palandri F, Castagnetti F, Iacobucci I, et al. The response to imatinib and interferon-alpha is more rapid than the response to imatinib alone: a retrospective analysis of 495 Philadelphia-positive chronic myeloid leukemia patients in early chronic phase. Haematologica. 2010; 95:1415-9.

68. Preudhomme C, Guilhot J, Nicolini FE, et al. Imatinib plus peginterferon alfa-2a in chronic myeloid leukemia. N Engl J Med. 2010; 363:2511-21.

69. Nicolini FE, Etienne G, Dubruille V, et al. Nilotinib and peginterferon alfa-2a for newly diagnosed chronic-phase chronic myeloid leukaemia (NiloPeg): a multicentre, non-randomised, open-label phase 2 study. Lancet Haematol. 2015; 2:e37-46.

70. Nicolini F, Etienne G, Huguet F, et al. (2018). Nilotinib versus nilotinib combined to pegylated-interferon alfa $2 \mathrm{a}$ in first-line chronic phase CML patients. Updated interim analysis of a phase III trial, the PETALS study. EHA 2018; Abstra PF378.

71. Yeung DT, Grigg AP, Shanmuganathan $\mathrm{N}$, et al. Combination of nilotinib and pegylated interferon Alfa-2b results in high molecular response rates in chronic phase CML: interim results of the ALLG CML 11 pinnacle study. Blood. 2018; 132:459.

72. Clark RE, Polydoros F, Apperley JF, et al. De-escalation of tyrosine kinase inhibitor dose in patients with chronic myeloid leukaemia with stable major molecular response (DESTINY): an interim analysis of a nonrandomised, phase 2 trial. Lancet Haematol. 2017; 4(7):e310-e316.

73. Rea D, Dulphy N, Henry G, et al. Low natural killer (NK) cell counts and functionality are associated with molecular relapse after imatinib discontinuation in patients (pts) with chronic phase (CP)-chronic myeloid leukemia (CML) with undetectable BCR-ABL transcripts for at least 2 years: preliminary results from Immunostim, on behalf of STIM Investigators. Blood. 2013; 122. Abstract 856.

74. Ilander MM, Olsson-Strömberg U, Lähteenmäki H, et al. Early disease relapse after tyrosine kinase inhibitor treatment discontinuation in CML is related both to low number and impaired function of NK-cells chronic myeloid leukemia: therapy. Blood. 2014; 124(21). Abstract 812. 